Pacific Journal of Mathematic 


\section{A CLASS OF MEASURE PRESERVING TRANSFORMATIONS}

\section{ChaRles Standish}

In this paper we shall consider the following class of transformations of the unit interval onto itself. Let $\pi$ be a permutation of the positive integers, that is, a one-to-one mapping of the positive integers onto themselves. Let $t(0 \leqq t \leqq 1)$ be represented in its dyadic expansion:

$$
t=\sum_{k=1}^{\infty} \varepsilon_{k}(t)
$$$$
\varepsilon_{k}=0 \text { or } 1 \text {. }
$$

Then we define

$$
T_{\pi}(t)=\sum_{k=1}^{\infty} \frac{\varepsilon_{\pi(k)}(t)}{2^{k}}
$$

$T_{\pi}(t)$ "shuffles" the digits in the dyadic expansion of $t$.

Our motivation in considering these transformations lies in the fact that they form a nontrivial class of measurable transformations with a simple intuitive interpretation and may be utilized to illustrate several of the concepts of ergodic theory.

\section{Measurability and ergodicity considerations.}

THEOREM 1.1. For every choice of $\pi, T_{\pi}$ is a measure preserving transformation.

Proof, Let $X_{i}(i=1,2 \cdots)$ be the space consisting of the two real numbers 0 and 1 endowed with a measure $m$ defined by $m(0)=1 / 2$ $m(1)=1 / 2$. Consider the product space $X=\prod_{i=1}^{\infty} X_{i}$ (where we omit those products for which all but a finite number of factors=1) and define the measure of a "rectangle" $\prod_{i=1}^{\infty} E_{i}, E_{i} \subset X_{i}$ by $\mu\left(\prod_{i=1}^{\infty} E_{i}\right)=\prod_{i=1}^{\infty} m\left(E_{i}\right)$ then it can be shown $[1$, p. 159] that the above measure is capable of extension to a measure on a $\sigma$ algebra of subsets containing the rectangles in such a fashion that the mapping

$$
\varphi: X \rightarrow[0,1]
$$

defined by

Received February 14, 1955, and in revised form August 4, 1955. This is a portion of a doctoral thesis written at Cornell University under the direction of Professor Mark Kac. The author wishes to thank Professor Kac for his guidance and assistance. 


$$
\varphi(x)=\sum_{i=1}^{\infty} \frac{x_{i}}{2^{i}}
$$$$
x_{i}=0 \text { or } 1
$$

sends the measurable subsets $S$ of $X$ onto the Lebesgue measurable sets of $[0,1]$, with $\mu(S)=$ Lebesgue measure of $\varphi(S)$. To demonstrate that $T_{\pi}$ is measure preserving we need only show that this is the case for product sets. But this is trivial since $T_{\pi}$ merely rearranges the factors in a product set and the measure of a product set is obviously invariant under a permutation of its factors.

THEOREM 1.2. A necessary and sufficient condition for $T_{\pi}(t)$ to be a metrically transitive (ergodic) transformation is that neither the permutation $\pi$ nor any of its iterates possess a fixed point.

Proof. For the definition of metric transitivity we refer to [2, p. 29]. We note that transformations satisfying the hypotheses of the theorem exist for example, $\pi(1)=2, \pi(2 k)=2 k+2, \pi(2 k+1)=\pi(2 k-1)$ consisting of a single infinite cycle is easily seen to have the desired properties. The demonstration of necessity is quite easy. Suppose that $\pi^{(n)}\left(\pi^{(n)}\right.$ denoting the $n$th iterate of $\left.\pi\right)$ has a fixed point $k_{0}$. That is, $\pi^{(n)}\left(k_{0}\right)=k_{0}$. Let $\pi\left(k_{0}\right)=k_{1}, \pi^{(2)}\left(k_{0}\right)=k_{2}, \cdots, \pi^{(n-1)}\left(k_{0}\right)=k_{n-1}$, then $\pi$ permutes the set $S:\left(k_{0}, k_{1}, \cdots, k_{n-1}\right)$. Consider the set

$$
\begin{aligned}
B=\prod_{k=1}^{\infty} A_{k} \quad A_{k} & =X_{k}, k \notin S \\
A_{k} & =1, \quad k \in S
\end{aligned}
$$

This set has measure $2^{-n}$ and is clearly invariant under $T_{\pi}$, but a transformation leaving a set of positive measure invariant is not metrically transitive. The sufficiency requires a more extended argument. It is our object to show that if $A$ and $\mathrm{B}$ are measurable sets with characteristic functions $\varphi_{A}$ and $\varphi_{B}$ respectively and if neither $\pi$ nor any of its iterates has a fixed point then

$$
\lim _{n \rightarrow \infty} \int_{X} \varphi_{A}\left[T_{\pi}^{n}(P)\right] \varphi_{B}(P) d \mu(P)=\left[\int_{X} \varphi_{A}(P) d \mu(P)\right]\left[\int_{X} \varphi_{B}(P) d \mu(P)\right]
$$

which is of course equivalent to

$$
\lim _{n \rightarrow \infty} \mu\left(T_{\pi}^{n}(A) \cap B\right)=\mu(A) \mu(B) .
$$

But this is the strong mixing property which implies metric transitivity and ergodicity [2, p. 36]. Our proof is in two parts. First we demonstrate the theorem for the case where $\varphi_{A}(P)$ and $\varphi_{B}(P)$ depend only upon a finite number of factors in the product space and then reduce 
the general case to this special one. Suppose now that $\varphi_{A}(P)$ and $\varphi_{B}(P)$ depend only on a finite number of factors, say

$$
\begin{aligned}
& \varphi_{A}(P)=\varphi_{A}\left(X_{k_{1}}, \cdots, X_{k_{n}}\right) \\
& \varphi_{B}(P)=\varphi_{B}\left(X_{k_{1}}, \cdots, X_{k_{n}}\right)
\end{aligned}
$$

Since neither $\pi$ nor any of its iterates has a fixed point there exists an $N$ such that for $n>N, \varphi_{A}\left(T_{\pi}^{n} P\right)$ does not depend on any of the factors $X_{k_{1}}, \cdots, X_{k_{n}}$, but for all such $n$

$$
\int \varphi_{A}\left(T^{n} P\right) \varphi_{B}(P) d \mu(P)=\left[\int \varphi_{A}(P) d \mu(P)\right]\left[\int \varphi_{B}(P) d \mu(P)\right],
$$

which proves our assertion for the special case. In the general case we observe that the characteristic function of any measurable set may be approximated in the $L^{1}$ sense arbitrarily closely by the characteristic function of a set depending on only a finite number of factors. (For a proof see $[2, \mathrm{pp} .4,57])$. Now given $\varphi_{A}(P), \varphi_{B}(P)$ and $\varepsilon>0$ we choose $\varphi_{A^{\prime}}(P)$ and $\varphi_{B^{\prime}}(P)$ such that

$$
\begin{aligned}
& \int\left|\varphi_{A}(P)-\varphi_{A^{\prime}}(P)\right| d \mu(P)<\varepsilon \\
& \int\left|\varphi_{B}(P)-\varphi_{B^{\prime}}(P)\right| d \mu(P)<\varepsilon
\end{aligned}
$$

where $\varphi_{A^{\prime}}$ and $\varphi_{B^{\prime}}$ depend on only a finite number of factors. Then

$$
\begin{aligned}
& \left|\int \varphi_{A}\left(T^{n} P\right) \varphi_{B}(P) d \mu(P)-\left[\int \varphi_{A}(P) d \mu(P)\right]\left[\int \varphi_{B}(P) d \mu(P)\right]\right| \\
\leqq & \left|\int \varphi_{A}\left(T^{n} P\right) \varphi_{B}(P) d \mu(P)-\int \varphi_{A^{\prime}}\left(T^{n} P\right) \varphi_{B^{\prime}}(P) d \mu(P)\right| \\
+ & \left|\left[\int \varphi_{A^{\prime}}(P) d \mu(P)\right]\left[\int \varphi_{B^{\prime}}(P) d \mu(P)\right]-\left[\int \varphi_{A}(P) d \mu(P)\right]\left[\int \varphi_{B}(P) d \mu(P)\right]\right| \\
+ & \left|\int \varphi_{A^{\prime}}\left(T_{\pi}^{n}(P)\right) \varphi_{B^{\prime}}(P) d \mu(P)-\left[\int \varphi_{A^{\prime}}(P) d \mu(P)\right]\left[\int \varphi_{B^{\prime}}(P) d \mu(P)\right]\right| .
\end{aligned}
$$

Our choice of $\varphi_{A^{\prime}}(P)$ and $\varphi_{B^{\prime}}(P)$ together with the measure preserving property of $T_{\pi}$ implies that (1.3) and (1.4) are each smaller than $2 \varepsilon$. Assuming (1.1) is true for the special case we have (1.5) $<\varepsilon$ for $n \geqq N(\varepsilon)$. Hence

$$
\lim _{n \rightarrow \infty} \mu\left(T_{\pi}^{n}(A) \cap B\right)=\mu(A) \cdot \mu(B),
$$

and the theorem is completely demonstrated. The techniques employed in the sufficiency proof were utilized for another purpose by Hopf [2, p. 57]. 
2. Convergence of certain series. We now turn our attention to an examination of the convergence of

$$
\sum_{k=0}^{\infty} \frac{f\left(T_{\pi}^{k} x\right)}{k}
$$

and

$$
\sum_{k=0}^{\infty} \frac{f\left(T_{\pi}^{\lambda_{k}} x\right)}{k}, \quad \begin{aligned}
& \lambda_{k} \text { a subsequence of } \\
& k \text { the positive integers. }
\end{aligned}
$$

The almost everywhere convergence of (2.1) yields a strengthened form of the Birkhoff ergodic theorem, for if (2.1) converges,

$$
\lim _{n \rightarrow \infty} n^{-1} \sum_{k=0}^{n} f\left(T_{\pi}^{k} x\right)=0 \text {. }
$$

This fact is an immediate consequence of the well known theorem that th convergence of $\sum_{n=1}^{\infty} c_{n} / n$ implies

$$
\lim _{n \rightarrow \infty}(1 / n) \sum_{i=1}^{n} c_{i}=0 .
$$

We are not able to establish convergence of (2.1) under the very mild restrictions placed on $f(x)$ in order for the ergodic theorem to hold. It is clear from the example $f(x)=$ constant that mere integrability of $f$ is not enough. Our consideration of series of the form (2.2) is motivated by studies of Kac [3, 4] regarding series of the form

$$
\sum_{k=0}^{\infty} \frac{f\left(T^{k} x\right)}{k}
$$

and

$$
\sum_{k=0}^{\infty} \frac{f\left(n_{k} x\right)}{k}, \quad n_{k} \text { integers, } n_{k} / n_{k+1}>q>1 .
$$

The techniques employed in the study of (2.4) can be made to yield some results concerning convergence of (2.2) although, as would be expected from the greater complexity of the transformations considered here, the results are not so sharp as those obtained by Kac.

Before stating and pproving the results of this section we must make some preliminary remarks. Our main tool will be the concept of quasi-orthogonal functions developed by Menchoff.

DEFINITION 2.1. A sequence of functions $\left\{f_{n}(x)\right\} n=1,2, \ldots$ is said to be quasi-orthogonal on a set $A$ if the quadratic form 


$$
\sum_{j, k=1}^{\infty} a_{j k} x_{j} x_{k} \quad a_{j k}=\int_{A} f_{j}(x) f_{k}(x) d x
$$

is bounded in Hilbert space, that is, there exists a constant $B$ independent of the $x_{j}$ such that

$$
\left|\sum_{j, k=1}^{\infty} a_{j k} x_{j} x_{k}\right| \leqq B \sum_{j=1}^{\infty} x_{j}^{2}
$$

Observe that an orthogonal sequence of functions is quasi-orthogonal since in this case $a_{j k}=\delta_{j k}$ and

$$
\sum_{j, k=1}^{\infty} a_{j k} x_{j} x_{k} \leqq \sum_{j=1}^{\infty} x_{j}^{2}
$$

The importance of quasi-orthogonality lies in the fact that Bessel's inequality holds in the sense that if

$$
\int_{A} F(x) f_{k}(x) d x=C_{k}
$$

then there exists a constant $D$ such that

$$
\int_{A} F^{2}(x) d x \geqq D \sum_{k=1}^{\infty} C_{k}^{2}
$$

thus every theorem on sequences of orthogonal functions which utilizes only Bessel's inequality in its proof is also valid for sequences of quasiorthogonal functions. In particular we shall need the fact that the following theorem of Menchoff [5, p. 236] is valid for quasi-orthogonal functions.

THEOREM 2.1. If $\left\{\theta_{k}(x)\right\}$ is a sequence of orthogonal functions then $\sum_{k=1}^{\infty} c_{k} \theta_{k}(x)$ converges almost everywhere provided

$$
\sum_{k=1}^{\infty} c_{k}^{2} \log ^{2} k<\infty
$$

DEFINITION 2.2. Let $t=\sum_{k=1}^{\infty} \frac{\varepsilon_{k}(t)}{2^{k}}$ then the Rademacher functions $r_{k}(t)$ are defined as follows

$$
r_{k}(t)=\left\{\begin{array}{r}
1 \text { if } \varepsilon_{k}(t)=0 \\
-1 \text { if } \varepsilon_{k}(t)=1
\end{array}\right.
$$

It is well known that the Rademacher functions form an incomplete orthonormal set on $[0,1]$. Moreover the Rademacher functions are sta- 
tistically independent, that is, denoting Lebesgue measure by $\mu$, we have

$$
\mu\left\{t \mid r_{1}(t)<a_{1}, \cdots, r_{n}(t)<a_{n}\right\}=\prod_{k=1}^{n} \mu\left\{t \mid r_{k}(t)<a_{k}\right\}
$$

DEFINITION 2.3. The sequence of functions $\left\{\psi_{l_{k}}(x)\right\}$ defined by

$$
\begin{gathered}
\psi_{0}(x)=1 \\
\psi_{n}(x)=r_{n_{1}}(x) \cdots r_{n_{k}}(x) \text { for } n=2^{n_{1}}+\cdots+2^{n_{k}}
\end{gathered}
$$

where the $r_{n_{i}}(x)$ are Rademacher functions, are called the Walsh functions. They form a complete orthonormal set (the completion of the Rademacher functions) and hence for every $f(x) \in L^{2}$ on $[0,1]$ the WalshFourier series

$$
\sum_{k=1}^{\infty} c_{k} \psi_{k}(x) \quad c_{k}=\int_{0}^{1} f(x) \psi_{k_{k}}(x) d x
$$

converges to $f(x)$ in the $L^{2}$ mean.

We are now ready to prove our theorems. In each of them it will be assumed that neither $\pi$ nor any of its iterates has a fixed point.

THEOREM 2.2. The series (2.1) either converges almost everywhere or diverges almost everywhere in $[0,1]$.

Proof. Denote by $C$ the set of points where the series converges. This set is invariant under $T_{\pi}$ but since $T_{\pi}$ is metrically transitive, either $C$ or its complement is a zero set.

THEOREM 2.3. Suppose $f(x)$ satisfies

$$
\begin{gathered}
\left|f(x)-f\left(x^{\prime}\right)\right| \leqq\left|x-x^{\prime}\right|^{\alpha} \\
\int_{0}^{1} f(x) d x=0
\end{gathered}
$$$$
\alpha>1 / 2,
$$

then $\sum_{k=1}^{\infty} c_{k} f\left(T_{\pi}^{k} x\right)$ converges almost everywhere provided

$$
\sum_{k=1}^{\infty} c_{k}^{2} \log ^{2} k<\infty
$$

Proof. We shall demonstrate that hypotheses (a) and (b) insure that the sequence $\left\{f\left(T_{\pi}^{k} x\right)\right\}$ is quasi-orthogonal. To do this we expand $f(x)$ in a Walsh-Fourier series 


$$
f(x) \sim \sum_{k=1}^{\infty} c_{k} \psi_{k}(x), \quad c_{k}=\int_{0}^{1} f(x) \psi_{k}(x) d x
$$

Then

$$
f\left(T_{\pi}^{\lambda} x\right) \sim \sum_{k=1}^{\infty} c_{k} \psi_{k}\left(T_{\pi}^{\lambda} x\right) .
$$

But the transformation $T_{\pi}$ permutes the Walsh functions. Hence

$$
f\left(T_{\pi}^{\lambda} x\right) \sim \sum_{k=1}^{\infty} c_{\varphi(\lambda, k)} \psi_{k}(x),
$$

where the $c_{\varphi(\lambda, k)}$ are the Walsh-Fourier coefficients of $f(x)$ in some order. It was shown by Fine $[6$, p. 394] that the conditions (a) on $f(x)$ is sufficient to insure the absolute convergence of the Walsh-Fourier development of $f(x)$. By Parseval's relation,

$$
\int_{0}^{1} f(x) f\left(T_{\pi}^{\lambda} x\right) d x=\sum_{k=1}^{\infty} c_{k} c_{\varphi(\lambda, k)} .
$$

Hence

$$
\sum_{\lambda=1}^{\infty}\left|\int_{0}^{1} f(x) f\left(T_{\pi}^{\lambda} x\right) d x\right| \leqq \sum_{\lambda=1}^{\infty}\left|\sum_{k=1}^{\infty} c_{k} c_{\varphi(\lambda, k)}\right| \leqq\left(\sum_{k=1}^{\infty}\left|c_{k}\right|\right)\left(\sum_{\lambda=1}^{\infty}\left|c_{\varphi(\lambda, k)}\right|\right) .
$$

Since the Walsh-Fourier series of $f(x)$ is absolutely convergent, its sum is independent of a rearrangement of its terms and

$$
\sum_{\lambda=1}^{\infty}\left|c_{\varphi(\lambda, k)}\right|<M \quad \text { (independent of } k \text { ) . }
$$

Thus

$$
\sum_{\lambda=1}^{\infty}\left|\int_{0}^{1} f(x) f\left(T_{\pi}^{\lambda} x\right) d x\right| \leqq M \sum_{k=1}^{\infty}\left|c_{k}\right| \leqq M^{2}
$$

Setting

$$
a_{j_{k}}=\int_{0}^{1} f(x) f\left(T_{\pi}^{j-k} x\right) d x
$$

we have

$$
\sum_{k=1}^{\infty}\left|a_{j k}\right| \leqq \sum_{k=-\infty}^{\infty}\left|\int_{0}^{1} f(x) f\left(T_{\pi}^{j-k} x\right) d x\right| \leqq 2 M^{2}
$$

By the triangular inequality and the trivial inequality

$$
\left|x_{j} x_{k}\right| \leqq x_{j}^{2}+x_{k}^{2}
$$

we have 


$$
\left|\sum_{k, j=1}^{\infty} a_{j k} x_{j} x_{k}\right| \leqq \sum_{j, k=1}^{\infty}\left|a_{j k} x_{j}\right|^{2}+\sum_{k, j=1}^{\infty}\left|a_{j k}\right| x_{k}^{2} \leqq 4 M^{2} \sum_{j=1}^{\infty} x_{j}^{2} .
$$

Therefore $\left\{f\left(T_{\pi}^{\lambda} x\right)\right\}$ is quasi-orthogonal, and applying Theorem 2.1 we have our result. For the case of convergence in the $L^{2}$ mean we have as an almost immediate consequence of Theorem 2.3 the following.

ThEOREM 2.4. If $\left\{f\left(T_{\pi}^{*} x\right)\right\}$ is quasi-orthogonal the series

$$
\sum_{k=1}^{\infty} c_{k} f\left(T_{\pi}^{k} x\right)
$$

converges in the mean of order 2 provided that

$$
\sum_{k=1}^{\infty} c_{k}^{2}<\infty
$$

Proof. we have

$$
\left|\int_{0}^{1}\left[\sum_{\lambda=m}^{\lambda=n} c_{\lambda} f\left(T_{\pi}^{\lambda} x\right)\right]^{2} d x\right|=\left|\sum_{j, k=m}^{n} c_{k} c_{j} \int_{0}^{1} f\left(T_{\pi}^{k} x\right) f\left(T_{\pi}^{j} x\right) d x\right| \leqq 4 M^{2} \sum_{k=m}^{n} c_{k}^{2} .
$$

But the convergence of $\sum_{k=1}^{\infty} c_{k}^{2}$ implies the last term is arbitrarily small for $m$ and $n$ large enough.

The smoothness restrictions on $f(x)$ in the above two theorems are heavy, and it might be conjectured that as in the case of the ergodic theorem only the restrictions that $f(x)$ be integrable (or of course square integrable in the case of Theorem 2.4) are necessary. We are unable to answer this for the case of pointwise convergence, but in the case of mean convergence the answer is in the negative. For Halmos has shown [7, pp. 286-88] that for an arbitrary metrically transitive transformation $T$ there functions in $L^{2}$ for which

$$
\sum_{k=1}^{\infty} \frac{f\left(T^{k} x\right)}{k}
$$

does not converge in the mean. His proof depends upon the spectral resolution of the unitary operator $U$ in the Hilbert space of $L^{2}$ functions defined by $U f(x)=f(T x)$.

We now turn our attention to the convergence of certain gap series of the form (2.2).

TheOREM 2.5. Suppose

$$
\left|f(x)-f\left(x^{\prime}\right)\right|<\left|x-x^{\prime}\right|^{a},
$$$$
0<\alpha<1
$$ 
( b )

$$
\int_{0}^{1} f(x) d x=0
$$

Then there exists a subsequence $\left\{\lambda_{k}\right\}$ of the positive integers (the subsequence depneding on the permutation $\pi$ but not on $f(x)$ ) such that

$$
\sum_{k=1}^{\infty} c_{k} f\left(T_{\pi}^{\lambda} k x\right)
$$

converges almost everywhere, provided that

$$
\sum_{k=1}^{\infty} c_{k}^{2}<\infty
$$

Proof. Utilizing a device of $\mathrm{Kac}[8, \mathrm{p} .652]$ we construct a sequence of functions $\left\{f_{k}(x)\right\}$ satisfying

$$
\begin{aligned}
\int_{0}^{1} f_{k}(x) d x=0, & \\
\sum_{k=1}^{\infty} c_{k}^{2} \int_{0}^{1} f_{k}^{2}(x) d x<\infty, & (0<\alpha<1), \\
\left|f(x)-f_{k}(x)\right|<B / 2^{\alpha k} &
\end{aligned}
$$

$\left\{f_{k}\left(T_{\pi}^{\lambda_{k} x} x\right)\right\}$ is a subsequence of independent functions.

The construction goes as follows. Divide the interval $[0,1]$ into $2^{k}$ parts. Let

$$
f_{k}(x)=\frac{1}{2^{k}} \int_{r / 2^{k}}^{r+1 / 2^{k}} f(t) d t, \frac{r}{2^{k}} \leqq x \leqq \frac{r+1}{2^{k}} \quad r=0,1, \cdots, 2^{k-1} .
$$

An easy calculation shows that (1) holds. Since

$$
\begin{gathered}
\sup \left|f_{k}(x)\right| \leqq \sup |f(x)| \leqq M \\
\sum_{k=1}^{\infty} c_{k}^{2} \int_{0}^{1}\left|f_{k}(x)\right|^{2} d x \leqq M^{2} \sum_{k=1}^{\infty} c_{k}^{2}
\end{gathered}
$$

which proves (2).

The construction of the $f_{k}(x)$ and hypothesis (a) imply (3). To prove (4) we need the following.

Lemma. Let $J$ be a fixed finite collection of integers. Let $\pi$ be the permutation defining $T$, then an integer $n_{0}$ can be so chosen that $\left\{\pi^{n}(1)\right.$, $\left.\cdots, \pi^{n}(k)\right\} \cap J=\phi$ if $n>n_{0} . \quad$ ( $\phi$ denotes the empty set.)

Proof. $\pi$ is a permutation without fixed points, hence there exists 
an integer $n_{1}$ such that for $n>n_{1}, \pi^{n}(1) \cap J=\phi$. Similarily there exist $n_{j}$ such that $\pi^{n}(j) \cap J=\phi, n>n_{j}, j=1, \cdots, k$. Now choose $n_{0}=\max \left(n_{1}\right.$, $\left.\cdots, n_{k}\right)$.

If $x=\sum_{k=1}^{\infty} \varepsilon_{k}(x) / 2^{k}, f_{k}(x)$ being a step function with jumps at points $r / 2^{k}$, is of the form $f_{k}(x)=F_{k}\left(\varepsilon_{1}, \varepsilon_{2}, \cdots, \varepsilon_{k}\right)$. Suppose we have chosen $\lambda_{1}, \cdots, \lambda_{n}$ so that $f_{1}\left(T^{\lambda_{1}} x\right) \cdots f_{n}\left(T^{\lambda_{n}} x\right)$ are independent. Now choose $\lambda_{n+1}$ such that $\pi^{\lambda_{n+1}}(j) \neq \pi^{\lambda_{k}}(m), \quad m<\lambda_{k}, k=1, \cdots, n, j=1, \cdots, n+1$. To see that this is possible take $J=\left\{\pi^{\lambda} k(m)\right\}, m<\lambda_{k}, k=1, \cdots, n$, and apply our lemma. Now

$$
f_{n+1}\left(T^{\lambda_{n+1} x}\right)=F_{n+1}\left(\varepsilon_{\pi^{n+1}}^{\lambda_{n+1}}(1), \cdots \varepsilon_{\pi}^{\lambda_{n+1}}(n+1)\right)
$$

is independent of $f_{k}\left(T^{\lambda_{k}} x\right), \lambda_{k}<n$, and (4) is proved. The series

$$
\sum_{k=1}^{\infty} c_{k} f_{k}\left(T^{\lambda_{k}} x\right)
$$

converges almost everywhere by the Kolmogoroff three series theorem. Since $\left|f_{k}\left(T^{\lambda_{k}} x\right)-f\left(T^{\lambda_{k}} x\right)\right|<B / 2^{\alpha k}, \sum_{k=1}^{\infty} c_{k} f\left(T^{\lambda_{k}} x\right)$ converges almost everywhere, and the proof of Theorem 2.5 is complete.

Upon specializing the permutation $\pi$ our results can be considerably strengthened. We illustrate by an example. Let $\pi=(\cdots 5312468 \cdots)$ Then if $f(x)$ satisfies the hypotheses of Theorem $2.5, \sum_{k=1}^{\infty} c_{k} f\left(T^{\lambda_{k}} x\right)$ converges for all sequences of integers $\left\{\lambda_{k}\right\}$ such that

$$
\lim _{k \rightarrow \infty} \frac{\lambda_{k+1}}{\lambda_{k}}>c>2
$$

One sees this by noting that for $k$ sufficiently large

$$
\lambda_{k+1}>\lambda_{k}+(k+2) \text {. }
$$

But then the sequence of functions $\left\{f_{k}\left(T^{\lambda_{k}} x\right)\right\}$ is independent. It might be conjectured that for a suitable permutation even the sequence $\left\{f_{k}\left(T_{\pi}^{k} x\right)\right\}$ is independent. This is not the case, however, by virtue of the following combinatorial lemma, which is of possible independent interest.

LEMmA. If $\pi$ is a permutation containing at least one infinite cycle then it is impossible that

$$
\pi(1), \pi^{2}(1), \pi^{2}(2), \cdots, \pi^{k}(1), \cdots \pi^{k}(k), \cdots
$$

are all distinct integers. 
Proof. Let $\left(\cdots k_{1}, k_{2}, \cdots\right)$ be an infinite cycle. Then it is impossible that $\pi\left(k_{\lambda}\right)<k_{\lambda}$ for all except a finite number of $\lambda$, since if this were the case then $\left\{k_{\lambda}\right\}$ for $\lambda$ sufficiently large would be a strictly decreasing infinite sequence of positive integers which is impossible. Hence there exist infinitely many $\lambda$ such that $\pi\left(k_{\lambda}\right)>k_{\lambda}$. Call this sequence again $\left\{k_{\lambda}\right\}$. If $\pi\left(k_{j}\right)=u_{j}$ then $u_{j}+1 \geqq k_{j}$ and $\pi^{u_{j}+1}\left(k_{j}\right)=\pi^{u}{ }^{u}\left(u_{j}\right)$

\section{A statistical remark.}

THEOREM 3.1. Given a transformation $T_{\pi}$ there exists a subsequence $\left\{\lambda_{k}\right\}$ of positive integers depending on $\pi$ but not on $f(x)$ such that if $f(x)$ satisfies a Lipschitz condition of order $\alpha$ and

$$
\lim _{n \rightarrow \infty} \frac{1}{n}\left\|\sum_{k=0}^{n} c_{k} f\left(T_{\pi^{\lambda}}^{\lambda_{k}}\right)\right\|^{2}=\sigma^{2} \neq 0 \quad \sum_{k=0}^{\infty} c_{k}^{2}<M,
$$

where

$$
\|\left. f\right|^{2}=\int_{0}^{1} f^{2} d x
$$

then

$$
\mu\left\{t \mid a<\frac{\sum_{k=0}^{n} c_{k} f\left(T_{\pi}^{\lambda_{k}} t\right)}{\sqrt{n}}<b\right\} \rightarrow \frac{1}{\sigma \sqrt{2 \pi}} \int_{a}^{b} \exp \left(-u^{2} / 2 \sigma^{2}\right) d u,
$$

that is, the sequence

$$
n^{-1 / 2} \sum_{k=0}^{n} c_{k} f\left(T_{\pi^{k}}^{\lambda_{k} t}\right)
$$

is asymptotically normally distributed.

Proof. By the proof of Theorem 2.5 we can find a sequence of statistically independent functions $\left\{f_{k}\left(T_{k}^{\lambda_{k}} x\right)\right\}$ such that

$$
\left\|f\left(T_{\pi^{k}}^{\lambda_{k}} t\right)-f_{k}\left(T_{\pi^{k}}^{\lambda_{k}} t\right)\right\| \leqq \frac{B}{2^{k x}} .
$$

Hence

$$
\left\|\sum_{k=0}^{n} c_{k} f\left(T_{k}^{\lambda} t\right)-\sum_{k=0}^{n} c_{k} f_{k}\left(T_{\pi^{k}}^{\lambda_{k}} t\right)\right\| \leqq B^{\prime} \sum_{k=0}^{n} \frac{1}{2^{k a}}<\text { constant } \quad\left(B^{\prime}=\max \left|c_{k}\right|+B\right) .
$$

Thus given $\varepsilon>0$

$$
\left\|\frac{\sum_{k=0}^{n} c_{k} f\left(T_{\pi}^{\lambda} k t\right)-\sum_{k=0}^{n} c_{k} f_{k}\left(T_{\pi^{k}}^{\lambda^{k} t}\right)}{\sqrt{n}}\right\|<\varepsilon
$$


if $n$ is sufficiently large. We may now proceed exactly as in the proof of a theorem of Kac [4, Theorem 1, pp. 41-42] with $f_{k}\left(T_{\pi}^{\lambda_{k}} x\right)$ playing the role of $\varphi_{k}\left(T_{\pi^{k}}^{\lambda^{k}} x\right)$ and with $2^{k} t$ replaced by $T_{\pi^{k}}^{\lambda^{k} t}$ to obtain our result.

\section{REFERENCES}

1. P. R. Halmos, Measure theory, New York, 1950.

2. E. Hopf, Ergodentheorie, New York, 1948.

3. M. Kac, Convergence of certain gap series, Ann. of Math., 44 (1943), 411-416.

4. _ On the distribution of values of sums of the type $f\left(2^{k} t\right)$, Ann. of Math., 47 (1946), 33-49.

5. M. Kac, R. Salem, and A. Zygmund, A gap theorem, Trans. Amer. Math. Soc., 63 (1948), 235-243.

6. N. J. Fine, On the Walsh Functions. Trans. Amer. Math. Soc., 65 (1949), 372-414.

7. P. R. Halmos, $A$ non-homogeneous ergodic theorem, Trans. Amer. Math. Soc., 66 (1949), 284-288.

8. M. Kac, Probability methods in some problems of analysis and number theory, Bull. Amer. Math. Soc., 55 (1949), 641-665.

\section{UNION COLLEGE}




\section{PACIFIC JOURNAL OF MATHEMATICS}

\section{EDITORS}

\author{
H. L. Royden \\ Stanford University \\ Stanford, California \\ E. Hewitt \\ University of Washington \\ Seattle 5 , Washington
}

R. P. Dilworth

California Institute of Technology

Pasadena 4, California

\author{
A. HorN* \\ University of California \\ Los Angeles 24, California
}

\section{ASSOCIATE EDITORS}

\author{
E. F. BECKENBACH \\ C. E. BURGESS \\ H. BUSEMANN \\ H. FEDERER
}

\author{
M. HALL \\ P. R. HALMOS \\ V. GANAPATHY IYER \\ R. D. JAMES
}

\author{
M. S. KNEBELMAN \\ I. NIVEN \\ T. G. OSTROM \\ M. M. SCHIFFER
}

J. J. STOKER

G. SZEKERES

F. WOLF

K. YOSIDA

\section{SPONSORS}

\author{
UNIVERSITY OF BRITISH COLUMBIA \\ CALIFORNIA INSTITUTE OF TECHNOLOGY \\ UNIVERSITY OF CALIFORNIA, BERKELEY \\ UNIVERSITY OF CALIFORNIA, DAVIS \\ UNIVERSITY OF CALIFORNIA, LOS ANGELES \\ UNIVERSITY OF CALIFORNIA, SANTA BARBARA \\ MONTANA STATE UNIVERSITY \\ UNIVERSITY OF NEVADA \\ OREGON STATE COLLEGE \\ UNIVERSITY OF OREGON \\ UNIVERSITY OF SOUTHERN CALIFORNIA
}

STANFORD RESEARCH INSTITUTE

STANFORD UNIVERSITY

UNIVERSITY OF UTAH

WASHINGTON STATE COLLEGE

UNIVERSITY OF WASHINGTON

AMERICAN MATHEMATICAL SOCIETY HUGHES AIRCRAFT COMPANY SHELL DEVELOPMENT COMPANY

Mathematical papers intended for publication in the Pacific Journal of Mathematics should be typewritten (double spaced), and the author should keep a complete copy. Manuscripts may be sent to any of the editors. Manuscripts intended for the outgoing editors should be sent to their successors. All other communications to the editors should be addressed to the managing editor, Alfred Horn at the University of California, Los Angeles 24, California.

50 reprints of each article are furnished free of charge; additional copies may be obtained at cost in multiples of 50 .

The Pacific Journal of Mathematics is published quarterly, in March, June, September, and December. The price per volume (4 numbers) is $\$ 12.00$; single issues, $\$ 3.50$. Back numbers are available. Special price to individual faculty members of supporting institutions and to individual members of the American Mathematical Society: $\$ 4.00$ per volume; single issues, $\$ 1.25$.

Subscriptions, orders for back numbers, and changes of address should be sent to Pacific Journal of Mathematics, c/o University of California Press, Berkeley 4, California.

Printed at Kokusai Bunken Insatsusha (International Academic Printing Co., Ltd.), No. 10, 1-chome, Fujimi-cho, Chiyoda-ku, Tokyo, Japan.

* During the absence of E. G. Straus.

PUBLISHED BY PACIFIC JOURNAL OF MATHEMATICS, A NON-PROFIT CORPORATION COPYRIGHT 1956 BY PACIFIC JOURNAL OF MATHEMATICS 


\section{Pacific Journal of Mathematics}

\section{Vol. 6, No. $3 \quad$ BadMonth, 1956}

Richard Arens and James Eells, Jr., On embedding uniform and topological spaces ............................................ 397

N. Aronszajn and Prom Panitchpakdi, Extension of uniformly continuous transformations and hyperconvex metric spaces .............. 405

Kai Lai Chung and Cyrus Derman, Non-recurrent random walks ........ 441

Harry Herbert Corson, III, On some special systems of equations . . . . . . . . . 449

Charles W. Curtis, On Lie algebras of algebraic linear transformations . . . 453

Isidore Heller, Neighbor relations on the convex of cyclic permutations . . . . 467

Solomon Leader, Convergence topologies for measures and the existence of transition probabilities..................................... 479

D. H. Lehmer, On certain character matrices ...................... 491

Michael Bahir Maschler, Minimal domains and their Bergman kernel function ........................................... 501

Wm. M. Myers, Functionals associated with a continuous transformation ................................... 517

Irving Reiner and Jonathan Dean Swift, Congruence subgroups of matrix groups ....................................... 529

Andrew Sobczyk, Simple families of lines ................... 541

Charles Standish, A class of measure preserving transformations ........ 553 Jeremiah Milton Stark, On distortion in pseudo-conformal mapping ..... 565 